\title{
Survival following gastrostomy insertion: are there differences in mortality according to referral indication?
}

\author{
M. Kurien ${ }^{1}$, J. S. Leeds ${ }^{1}$, H. E. Robson ${ }^{1}$, G. James ${ }^{2}$, B. Hoeroldt ${ }^{3}$, K. Dear ${ }^{4}$, K. Kapur ${ }^{5}$, J. Grant ${ }^{1}$, \\ M. E. McAlindon ${ }^{1}$ and D. S. Sanders ${ }^{1}$ \\ ${ }^{1}$ Department of Gastroenterology, Royal Hallamshire Hospital, Sheffield, ${ }^{2}$ Department of Gastroenterology, Doncaster \\ Royal Infirmary, Doncaster, ${ }^{3}$ Department of Gastroenterology, Rotherham District General Hospital, Rotherham, \\ ${ }^{4}$ Department of Gastroenterology, Chesterfield Royal Hospital, Chesterfield and ${ }^{5}$ Department of Gastroenterology, \\ Barnsley District General Hospital, Barnsley, UK
}

Previous studies have demonstrated that certain subgroups of patients have a worse prognosis following gastrostomy insertion (e.g. Dementia). However, these studies have often been retrospective and sample sizes have been small. We wished to examine survival following gastrostomy insertion with particular reference to the referral indication.

Gastrostomy insertions were examined from six hospitals in South Yorkshire and Derbyshire between January 2004 and September 2010. Data was collected prospectively from two centres $(n=1004)$ and from four centres retrospectively $(n=679)$. Age, gender, referral indication, comorbidity, biochemical profile and whether they were alive at 30 days was recorded for each case. Referral indication was divided into five subcategories; dysphagic stroke, neurological, nasopharyngeal cancer, cognitive impairment and other. Analysis for factors associated with death at 30 days was performed.

1651 patients were included in the study and the lowest 30-day mortality was found in the nasopharyngeal cancer cohort at $36 / 568$ $(6.3 \%)$. With respect to the other referral indications, 30-day mortality was $2 / 14(14.3 \%$, OR $2.5,0.5-11.4, p=n s)$ in cognitive impairment, 91/488 $(18.6 \%$, OR 3.4, 2.3-5.1, $p<0.001)$ in dysphagic stroke, 36/324 (11.1\%, OR 1.8, 1.1-3.0, $p=0.012)$ in neurological disease and $28 / 257(10.9 \%$, OR $1.8,1.1-3.0, p=0.024)$ in the other category. Mortality at 30 days was also associated with increasing age $>60$ (OR 3.1, 2.1-4.6, $p<0.001)$, reducing serum albumin $(p<0.001)$, cardiac comorbidity (OR 2.1, 1.3-3.4, $p=0.0023$ ), renal comorbidity (OR 2.1, 1.3-3.4, $p=0.004$ ) and intercurrent infection (OR 3.0, 1.7-5.1, $p<0.001)$. At multivariate analysis only age and albumin were independent predictors of death at 30 days (both $p<0.01$ ).

This is the largest UK dataset to assess outcomes following gastrostomy insertion. Indication for gastrostomy does have a bearing on mortality at 30 days with the lowest rates being found in patients with nasopharyngeal cancer. Our observations regarding mortality in these subgroups may be informative for clinicians, patients and relatives when making decisions around gastrostomy insertion. 